\title{
Antropologia Medica como Propedeutica de la Bioetica: Una Propuesta Curricular*
}

\author{
Medical Anthropology as Basic Discipline \\ for the Teaching of Bioethics
}

Miguel Kotton,1

PAIABRASCIAVES:

Antropología médica;

- Bioética;

Vulnerabilidad.

\section{KEY-WORDS:}

-Medical anthropology, - Bioethics,

- Vulnerability.

- Versión ampliada de una ponencia presentuda al II Congreso Anuml, Asociación de Humbidades Médicas. Stunnsén, Inglaterra, 19-21 de Julio 2004. 'Profesor Titular, Depto. de Biostica y Humunidades médicas. Escuela de Salud Pública Universidad de Chile. Miembro

Directivo, Red Latinoamericana de Bioética-UNNESCO, Chile.

\begin{abstract}
A Bioética refletc sobre os valores comprometidos nas práticas biomédicas, ponderando e determinando-os do modo mais correto possivel, em concordância com o caráter disciplinar que seu discurso deve ter: A reflexāo ética é fundamentalmente prescritiva, requcrendo uma análise prévia quc descreva e cspecifique os valores envolvidos nas práticas biomédicas, um trabalho propedéutico que se sugere encarregar à Antropologia Médica. A Antropologia Médica comeca por reconhecer vivencias de vulnerabilidade e de "enfermabilidade" do ser humano quc constitui a base da saúde pública empenhada cm atenuar essa vivência de fragilidado biologica. Quando cai enfermo - homo infirmus -, o sujeito deve scr entendido antropologicamente corpo vivo - organismo lesionado - e corpo vivido - existência ameaçada ou encerrada , mas também como pessoa com a cxpectativa de poder ser curada. Como tal autoriza ao terapeuta para cuidar e tratar o dano orgânico como também o declínio espiritual, gerando-se a base para o pacto fiduciário que fundamenta o encontro clínico. É apresentado um resumo de temas das dimensócs antropológicas e médicas que servem de fundamento ans estudos da Medicina.
\end{abstract}

RESUMO

\begin{abstract}
Bioethics is concerned with the values embedded in biomedical practices, deliberating and assigning these values according to the requirements of strict scholarly reflection. Ethical thought is basically prescriptive, requiring a previous analysis based on the description and specifications of the values involved. It is suggested in this paper that medical anthropology can act as a propedeutic discipline, capablc of recognizing the essential human attributes and experiences of vulnerability and disease-proneness. When an individual becomes ill, he suffers both damage to the living body and to the lived body, for disease threatens function as well as existential being. Seeking thcrapcutic assistance means that suffering disease is accompanicd by the hope of recovery, thus setting the basis for the fiduciary covenant that underlies the clinical encounter: Anthropology shows the physician-patient relationship to be more than just a tcchnical or contractual one, for the patient brings his cxistential anguish to the encountcr, and the physician is cxpected to attend the sick living body as well as its lived dimension.
\end{abstract}




\section{EL ESTATUS DISCIPLINAR DE LA BIOÉTICA}

Hay ciertas discrepancias acerca del estatus disciplinar de la bioética. Desde las escuelas pragmáticas se tiende a pensarla como una estrategia para solucionar problemas clínicos, ${ }^{1}$ que no requiere una estructuración básica más allá de aplicar técnicas de comunicación, decisión, evaluación de competencia mental, representatividad. Veta pragmática adicional ha sido el enfrentamiento de situaciones legales y judiciales: la figura del suicidio médicamente asistido no es otra cosa que una protección legal que soslaya la discusión ética de fondo, algo similar ocurre con la doctrina del doble efecto. Se da, por otro lado, el rechazo iracundo de la reflexión bioética acusándola de propiciar el análisis de temas supuestamente indiscutibles como el aborto procurado, la eutanasia, el homicidio de embriones. $^{2}$

Frente a estas críticas, la bioética se desenvuelve de un modo que substancial y sociológicamente corresponde a una disciplina ( discipere: analizar para entender). En lo substancial, tiene un temario propio y definido -las prácticas biomédicas -, una manera propia de abordarlas -ética aplicada -, una meta -asignar valores y rescatar derechos e intereses de los desaventajados - Según algunos tiene también un método, pero la diversidad de enfoques hace improbable que se pueda identificar una metodología propiamente bioética, ${ }^{3}$ como tampoco se logra identificarle un modo epistemológico y una enseñanza curricular característicos.

Como fenómeno social, la bioética se comporta sin duda como una disciplina: tiene presencia académica consistente en cursos regulares, departamentos, cuerpos docentes. Cuenta con actividades científicas en forma de congresos nacionales e internacionales, y una profusa lista de publicaciones periódicas, libros, actas, declaraciones. Y, loable o no, el carácter disciplinar de la bioética se confirma con su entrada al mercado como una experticia negociable.

La balanza se inclina en favor de entender la bioética como una disciplina estructurada, pero este reconocimiento conlleva responsabilidades no siempre reconocidas. Es excesivamente liviano iniciar el discurso diciendo que la bioética es multidisciplinaria; lo es, efectivamente, pero ello no hace sino reconocer que la cultivan personas de muy diversa filiación profesional, lo cual es obvio pero no señala algún atributo esencial de la bioética. Alternativamente se habla de que es interdisciplinaria, lo cual es una constatación pero no una caracterización propia, pues muchos problemas complejos son abordados por la conjunción de especialistas con perspectivas disciplinares diversas. Más apropiado es hablar de transdisciplinariedad, porque éste sí es un atributo singular, la bioética teniendo por objetivo básico insertarse en otras disciplinas como medicina, ecología, biología, agronomía, etc.; y, a la inversa, lo que se dice en filosofía, sociología, genética o economía tiene que ser recepcionado por una bioética esclarecida. En la transdisciplinariedad está el reconocimiento que la bioética es disciplina entre disciplinas.

Las observaciones introductorias presentadas pretenden reforzar la solidez conceptual de la bioética, que ha de ser respetada y enriquecida por sus representantes y cultores. Como toda disciplina, la bioética comienza por reconocer el terreno común de quienes la desarrollan, aceptando la existencia de una comunidad bioética que comparte valores aunque cada uno los jerarquice de otro modo. Una definición de bioética la sindica como la disciplina dedicada a la "inclusión de los valores en la toma de decisiones sanitarias", ${ }^{4}$ una propuesta implausible por cuanto la medicina ha estado ínsita de valores desde illo tempore, cuando aun era protomedicina chamánica. Más propio es pensar la bioética enfrentando las prácticas médicas a objeto de asignar adecuadamente sus valores que, aunque siempre presentes, han sido administrados en forma éticamente falente. No es que la bioética haya introducido, por ejemplo, el tema de la autonomía en la relación médico-paciente, pero sí desarrolló la idea de caducar el paternalismo autoritario y entregarle al paciente el manejo de su libertad de decisión. Es preciso alcanzar un esclarecimiento de valores que hasta ahora ha sido insuficiente y polémico, como lo ilustra el escándalo académico desencadenado en torno al concepto de dignidad, ${ }^{5}$ que por otra parte fue declarado como un principio de la bioética por el principialismo europeo. ${ }^{6}$ Es aquí donde se inserta la idea de una propedéutica de valores que interesan a la bioética, y la sugerencia de asignar esta tarea a la antropología médica.

En el intento de encontrar fundamentos más sólidos a los elementos argumentativos de la bioética, de rastrear su proveniencia y poderlos someter a un escrutinio en alguna medida objetivo, se ofrece la antropología y, más específicamente, la antropología médica, como disciplina fundante al menos para la bioética preocupada de la medicina latu sensu, es decir, tanto de la salud pública como de la práctica médica terapéutica. La antropología general reconoce cuatro subdisciplinas: etnología, lingüística, arqueología y antropología física, ${ }^{7}$ y las antropologías especiales también tienen diversos modos de cultivo. La principal perspectiva para la antropología médica ha sido hasta ahora la etnografía médica que recolecta información sobre los métodos de sanación de diversos grupos sociales, lo cual puede tener gran interés en las interacciones de la medicina pública con enfoques médico-culturales diversos. Mas, para las prácticas médicas y para la bioética que pudiese ser denominada occidental, interesan los atributos 
humanos que están comprometidos en la enfermedad, en las prácticas de sanación, en la medicina preventiva, así como el posible impacto de las biotecnociencias sobre lo que hasta ahora son consideradas las características antropológicas fundamentales e inamovibles del ser humano.

\section{Vulnerabilidad y enfermabilidad}

Desde el Renacimiento viene estableciéndose como atributo antropológico esencial la vulnerabilidad del individuo, producto de la falta de instintos y la consiguiente necesidad de construirse un proyecto de vida en permanente riesgo de fracasar y que inexorablemente quedará clausurado por la muerte. Uno de las probables causas de interferencia o claudicación es la enfermedad, que reduce capacidades y estrecha los rangos de autonomía efectiva, produciendo una pérdida de empoderamiento social. Tal como el individuo tiene consciencia de su vulnerabilidad y de su mortalidad, así también sabe de su enfermabilidad, lo cual tiene profunda influencia sobre su disciplinada aceptación de la medicina preventiva con sus propuestas de acotar este temor a enfermar mediante medidas de resguardo. ${ }^{8}$ Todo el afán de la medicina preventiva consiste en ofrecer una reducción y algún grado de control sobre la enfermabilidad, los individuos cooperando con estos programas desde su vivencia de vulnerables a enfermar, y el deseo de apagar esa vulnerabilidad; de manera que la efectividad de la salud pública dependerá de su capacidad de paliar la enfermabilidad, la bioética cultivando desde la protección, por ejemplo, los valores que en estas acciones de salud pública se presentan. 9,10

La enfermabilidad no sería un vector antropológico esencial si no lo fuese también el estado de enfermedad que aquella anuncia. La enfermedad es una experiencia vital, no un mero estado biológico, ganando espesor existencial si se la entiende, análogamente al ser humano sano, como un suceso biográfico mucho más complejo que un desperfecto del organismo. Recurriendo a un pensamiento medieval renovado por E. Strauss, el ser humano es aquel que se sostiene con sus pies sobre terra firme, gracias al desarrollo de la bipedalidad que le permitió el desarrollo de cerebración, lenguaje, visión binocular, manualidad, elementos todos con los que forja su existencia. ${ }^{11}$ La delicada inestabilidad de estas funciones explica su fragilidad, la vivencia de vulnerabilidad y la eventual pérdida del anclaje en terra firme, para caer en la enfermedad, el estado de homo infirmo. Entender enfermedad como pérdida de anclaje en tierra firme indica que el compromiso orgánico se acompaña de un desorden existencial más vasto, imposible de ser entendido en las categorías deterministas en que opera la medicina científico-natural. ${ }^{12}$

\section{Cuerpo vivo y cuerpo vivido}

Sucintamente fue esto captado por Merleau-Ponty, poco después por Plügge, ${ }^{13,14}$ al hablar de cuerpo vivo y cuerpo vivido, o Körper y Leib, una distinción que en absoluto puede comparase con la substancial - en res cogitans y res extensa- que hiciese Descartes. Aquí se trata de una dimensión antropológica de saberse cuerpo, de tener pero también ser un cuerpo-cuerpo vivido-, una consciencia de sí exacerbada en la enfermedad como cuerpo lesionado-cuerpo vivido enfermo- que desencadena la vivencia de todas las dimensiones del enfermar: sufrimiento, impedimentos, discapacidades, amenazas. El ser humano, como todos los seres vivos, es ampliamente descrito como un ente autopoiético -genera sus propios componentes para poder sustentar los procesos con los cuales genera estos componentes, y así recursivamente-. Las máquinas, que no son autopoiéticas, reciben su impulso de funcionamiento desde el exterior, por lo que son heteropoiéticas. Sería un error, sin embargo, considerar que la autopoiesis lesionada -el individuo enfermo-, al ser incapaz de mantenerse se convierte en heteropoiético, pues eso significaría que entrega su autonomía a la autoridad paternalista del terapeuta que se convierte en técnico reparador de un organismo disfuncionante. Más adecuado es reconocer en la autopoiesis claudicante una situación de impoiesis incapacidad de realizar el ciclo autopoiético pero sin haber perdido la autonomía de tomar las decisiones necesarias para su recuperación. El concepto antropológico del cuerpo vivido enfermo reconoce la impotencia restaurativa del enfermo, mas no por ello acepta que se vuelva disautónomo. Dicho de otro modo, la autonomía del enfermo, que es tan cara a la bioética, se fundamenta en el concepto antropológico del cuerpo vivido que enferma, de la autopoiesis que es anulada por una impoiesis paralizante pero deseosa de recuperación.

Para la bioética es importante recoger la vivencia de enfermedad en su proyección existencial, a fin de que la medicina científico-natural reconozca las valoraciones que esta vivencia da a la enfermedad, matizando y modificando el proceso de decisión informada. Solo a través del eje antropológico-bioético-médico es comprensible que el paciente pueda tomar decisiones que contravienen las recomendaciones médicas o parecen ir en contra de sus propios intereses.

\section{El encuentro clínico como encargo terapéutico}

La vivencia de enfermedad se acompaña por definición de la incapacidad de controlar y revertir el proceso por resorte a recursos propios. La enfermedad se caracteriza por su 
incapacidad de autoreparación, impulsando la búsqueda de ayuda externa a manos de un experto terapeuta. Esta búsqueda de ayuda está inspirada en la vivencia de que la enfermedad es removible, que el enfermo se siente sanable. La confianza en la sanabilidad y en el sanador constituye modos antropológicos de vivir la enfermedad, porque solo el hombre puede imaginar la curación y crear la figura del sanador, momentos que tienen una nítida traducción bioética, ante todo ilustrando que la búsqueda del terapeuta proviene del paciente que encarga o comisiona la participación de quien le ayudará a curar. La insistencia de la bioética por atribuirle a la relación médico-paciente un carácter fiduciario ancla en las constantes antropológicas del enfermar humano, de la conciencia de curabilidad por intermedio de ayuda externa, y por el encargo terapéutico que el enfermo entrega al sanador, constituyéndose así el encuentro clínico. Un terapeuta comisionado no está autorizado a tomar actitudes paternalistas en que los intereses del paciente son desatendidos.

Reconociendo esta génesis del encuentro con el terapeuta queda delineado que también éste tiene una estructura antropológica propia, consistente en 3 facetas: 1 ) Ser depositario de la confianza del paciente que lo motiva a iniciar el encuentro clínico; 2) Siendo el llamado del enfermo dirigido en primera término hacia la ayuda terapéutica, tiene el sanador la obligación de las prestaciones técnicas en su campo de experticia; 3 ) Siendo que el enfermo acude al encuentro con el médico, portando tanto su cuerpo vivo como el cuerpo vivido, el terapeuta enfrenta un enfermo que es paciente (= passio, el que sufre, el que está afectado), al que ha de acoger en su dolencia orgánica y en su desmedro existencial.

Uniendo los elementos hasta aquí descritos se constituye el encuentro clínico al cual el enfermo ingresa con cuatro elementos: cuerpo vivo lesionado, cuerpo vivido existencialmente claudicante y preocupado, confianza en la posibilidad de curación y encargo a un terapeuta considerado competente para hacerlo. El terapeuta, a su vez, acepta que recibe un encargo terapéutico que lo compromete a respetar la autonomía y los intereses del paciente, acercándose con competencia técnica y con la apertura de incorporar en las decisiones que se tomen la dimensión existencial que se encuentra comprometida por la enfermedad.

Se constituye así, desde los atributos antropológicos descritos, el encuentro clínico participativo, que no queda solo en el contrato o en el cumplimiento técnico según el determinismo de una medicina científico-natural, ni tampoco en el cumplimiento de un principialismo normativo. Adquiere fundamento antropológico la propuesta de una relación fiduciaria, de un pacto de confianza, como lo resaltaran desde los inicios de la bioética P. Ramsey y también E. Pellegrino.

La medicina clínica recibe su legitimidad moral del hecho de ser comisionada por el paciente, nunca pudiendo ser impuesta por iniciativa propia o por una instancia externa, pues cuando lo ha hecho-esterilizaciones mandatorias, internaciones hospitalarias forzadas-, ha cometido transgresiones éticas de envergadura. En salud pública sucede algo similar, en el sentido que la imposición de medidas preventivas requiere legitimarse por una aprobación o al menos por la ausencia de rechazo. Y esta aprobación pasa, a su vez, por la vivencia de enfermabilidad y la promesa de la salud pública de contar con medidas efectivas para evitar enfermedades.

Antes que se inicien acciones médicas preventivas o terapéuticas y antes que sean éticamene evaluadas, ya existen rasgos antropológicos que introducen el factor valorativo en la posibilidad de enfermar y en el enfermar mismo, así como la fe en expectativas de curación, y la confianza en el acto terapéutico. La bioética ha realizado ingentes esfuerzos por clarificar los valores que están en juego en todos estos momentos de la enfermedad humana, pero la fundamentación de estos valores le viene dada por la antropología médica que los detecta con anterioridad y los pone a su disposición para mejorar el desempeño de la práctica médica o techné iatriké.

\section{Límites antropológicos}

Las capacidades de la tecnociencia por modificar procesos biológicos han suscitado la interrogante acaso existe alguna limitación a las interferencias que el ser humano puede llevar a efecto en el mundo de los sers vivos. Dada la larga historia de alteraciones programadas en la agricultura y en lo pecuario, no hay mayor fundamento para negarles a las técnicas reproductivas y a las genéticas el hacer uso de sus capacidades para transformar el entorno vegetal y animal en beneficio de la humanidad. No obstante, el aprendiz de brujo ha invadido su propia biología y se dispone a modificar el genoma humano con consecuencias imprevisibles e irreversibles. Hasta ahora, la reflexión bioética ha sido incapaz de elaborar un pensamiento coherente con el cual sólidamente rechazar o aceptar la manipulación genética humana, incluyendo la clonación reproductiva. La antropología pregunta acaso el ser humano puede transformarse en su propio arquitecto como si fuese naturaleza maleable, o si obedece a una ética trascendental, en el sentido de fundamental, que lo conmina a no alterar lo esencialmente humano por cuanto perdería todo punto arquimédico desde donde dialogar consigo mismo y, sobre todo, entenderse como agente moral. ${ }^{15}$ Pregunta específica a la antropología médi- 
ca es acaso la normalidad de la salud puede ser artificialmente traspasada, generando seres humanos para quienes se hace posible romper los límites fisiológicos y de longevidad naturalmente dados. La antropología médica debe participar críticamente en el debate por la transformación de "<tratamientos médicos> en < tratamientos en cuidados de salud> (realizados para cumplir deseos relacionados con los aspectos biológicos de la vida)."16 Es evidente que la antropología médica debe tomar parte en la discusión que atañe las constantes de lo humano y su posible modificación.

\section{El rol curricular de la antropología médica}

La enseñanza de la bioética se ha incroporado definitivamente a la formación de profesionales biomédicos, así como de otras discplinas - agronomia, derecho, economia-. No existe, sin embargo, acuerdo alguno sobre la forma de abordar esta enseñanza, ni sobre las metas a proponer. La experiencia de varios años parece ratificar que la bioética curricular difícilmente es capaz de modificar actitudes, habiendo quienes dudan que la sensibilidad moral de los profesionales sea adecuadamente cultivada através de la enseñanza programada. ${ }^{17}$ Este escepticismo concordaría con la teoría kohlbergiana según la cual la madurez moral se alcanza en la adolescencia, y coincide con los estudios indicadores de que la preocupación ética decrece en el transcurso de los estudios médicos. 18

La frustración va acompañada del indisminuido convencimiento de la necesidad por mantener la bioética en los curriculos biomédicos. Posiblemente deba abandonarse los esfuerzos por crear determinadas actitudes porque, como dice Aristóteles a proposito de las virtudes, son hábito pero también disposición, y la disposición no es moldeable. Tal como la anatomia ilumina la estructura del cuerpo humano, tal vez la bioética clarifique y ayude a reflexionar sobre la estructura moral y los valores involucrados en la vulnerabilidad y la enfermabilidad, en el ser humano enfermo, sanable y entrelazado con el terapeuta en una relación fiduciaria que se despliega en el encuentro clínico. Con lo cual se está hablando el lenguaje de la antropologia médica, sustentando la propuesta que esta disciplina describe los atributos humanos pertinentes y los valores que concitan, para of recer un panorama descriptivo con el cual la bioética realice sus propuestas prescriptivas.

La literatura reconoce intentos previos de interacción entre antropología y bioética, pero con un punto de inflexión diferente al aquí presentado. Lamentando que la bioética desatienda las variantes de perspectivas éticas que se dan en diversas culturas, y la excesiva confianza puesta en el senti- do común como fundamento moral generalizado, se sostiene que "[E]n tanto los antropólogos de la medicina y bioética tienen consciencia de la presencia contemporánea de tradiciones terapéuticas distintas entre si, no han prestado los bioeticistas suficiente atención a esta creciente masa de producción académica."19 La antropología precisamente ha de situarse en un plano más fundamental, donde describe los atributos característicos de lo humano, de los cuales emergen valores generales como salud y sanación, disvalores como enfermedad, discapacidad, temor a enfermar. Acaso estos valores básicos se traducen en más o menos autonomia, paternalismo, compasión o benevolencia, son aspectos de la medicina que la bioética estudia, compara, reflexiona y propone.Una confusión similar ocurre en el principialismo europeo que describe vulnerabilidad, dignidad, integridad y autonomia como principios bioéticos, cuando en rigor se trata de constantes antropológicas en las cuales no está inscrita de inicio una normativa,ética, si bien se insinúa por la convocatoria a proteger estos atributos. 20

La propuesta de orientar la enseñanza a clarificar valores no es nueva, pero se ha presentado como "un esfuerzo curricular amplio destinado a mejorar la comprensión del médico de sus propios valores"21 utilizando "una técnica habitual de develar los valores del estudiante en su enfoque de estudios de casos". ${ }^{21}$ Este enfoque aproxima la enseñanza a una exploración psicológica de los futuros agentes sanitarios, en tanto lo aqui planteado es que la práctica biomédica encarna valores que anclan en atributos definitorios de lo humano, de manera que el esclarecimiento es una labor más bien antropológica.

En refuerzo de la inicialmente defendida proposición de entender la bioética formalmente como una disciplina, y de cultivar y enseñarla con el rigor correspondiente, se desarrolla un curriculum propedéutico de la antropología como una búsqueda estricta y fundamentada de los atributos humanos y los valores que protagonizan el temor de enfermar, la realidad existencial de estar enfermo, las actitudes y los protagonistas de prevención y curación (ver Anexos). La bioética se hace cargo de esclarecer y atribuir estos valores en el contexto de las prácticas biomédicas.

\section{Anexos}

El cuadro ilustra las dos vertientes de la antropología médica, la enfermabilidad que desde la vulnerabilidad lleva a la salud pública, en tanto que por la vivencia de enfermedad remata en el encuentro terapéutico. El temario antropológico (gris) sirve de base al temario bioético (punteado), ambos coincidiendo en el locus del encuentro clínico terapéutico. 


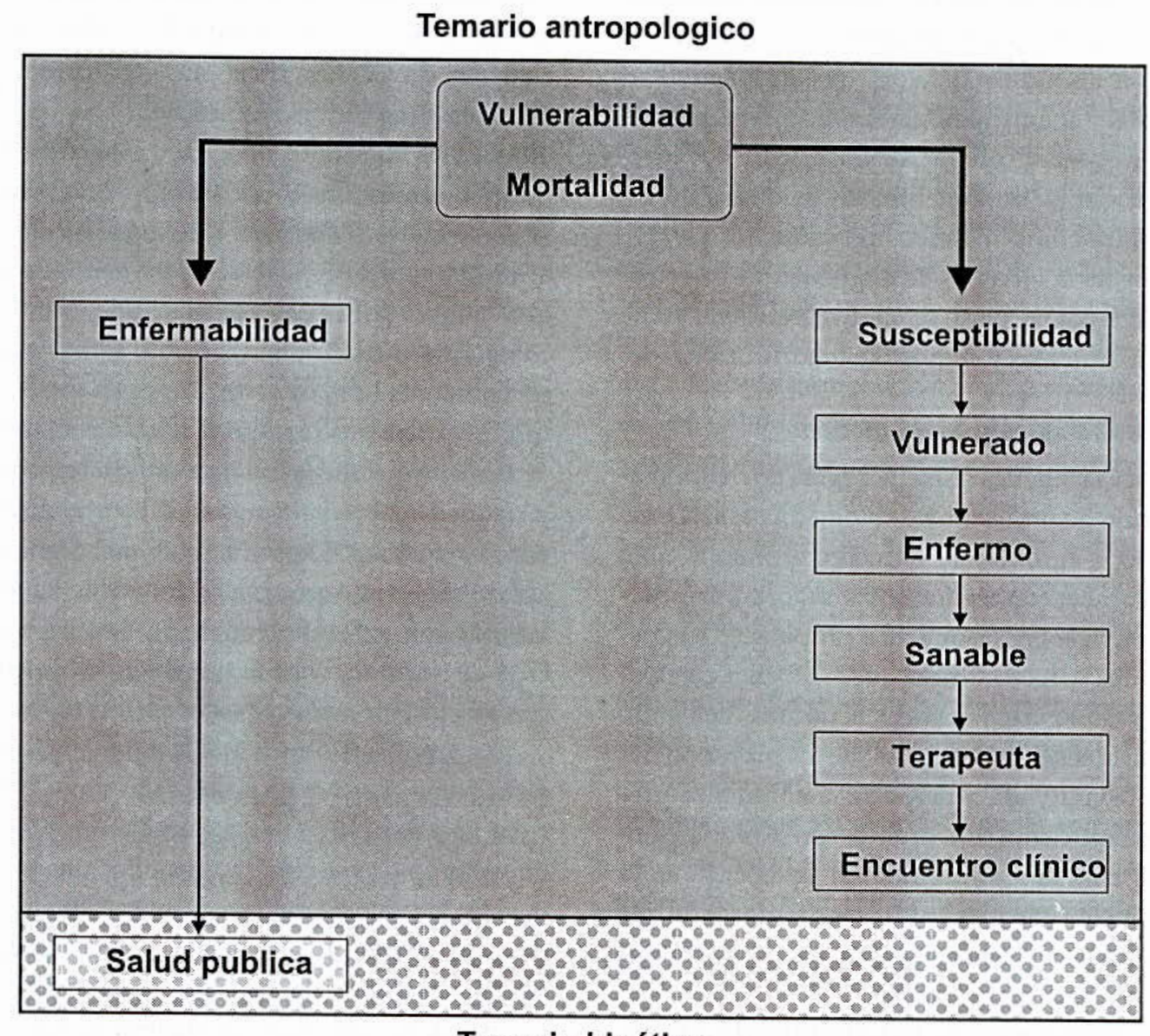

Temario bioético

Malla curricular de antropología médica como propedéutica de la bioética ${ }^{22}$

Introducción a la antropología

Antropología médica

Antropología etnomédica

Antropología hermenéutica o interpretativa

El cuerpo humano

Dolor y sufrimiento

Enfermedad y vivencia de enfermar

El terapeuta y el encuentro clínico

Enfermabilidad y salud pública

Modificaciones biomédicas de constantes antropológicas

\section{REFERENCIAS}

1. Iltis AS. Principlism and Casuistry. J Med Philosophy 2000 ; 25 : 271-284.

2. Smith WJ. Is bioethics ethical ? The Weekly Standard, Abril 3, 2000 .
3. Beauchamp TL. The Nature of Applied Ethics. In: Frey RG \& Wellman CH. A Companion to Applied Ethics. Oxford, Blackwell, 2003 : 1-16.

4. Gracia D. El qué y el porqué de la bioética. Cuadernos del Programa Regional de Bioética $1995 ; 1$ :35-53.

5. Macklin R. Dignity is a useless concept. Brit Med J 2003; 1419-1420

6. Rendtorff JD \& Kemp P. Basic Ethical Principles in European Bioethics and Biolaw. Barcelona, Instituto Borja de Bioética 2000.

7. Adams WY. Las raíces filosóficas de la antropología. Madrid: Trotta; 2003.

8. Laín Entralgo P. Antropología médica. Madrid: Salvat Ed; 1985.

9. Kottow M. Sanitary justice in scarcity. Cad. Saúde Pública 1999; 15(Sup. 1): 43-50.

10. Schramm FR, Kottow M. Principios bioéticos en salud pública: limitaciones y propuestas. Cad. Saúde Pública 2001; 17: 949-956. 
11. Spicker SF, Pellegrino ED. Embodiment and Rehabilitation. J Med Philosophy 1984; 9:1-120.

12. Spicker SF. Terra firma and Infirma Species: From Medical Philosophical Anthrpology to Philosophy of Medicine. J Med Philosophy 1976; 1: 104-135.

13. Merleau-Ponty M. Phénoménologie de la Perception. Paris: Gallimard; 1945.

14. Plügge $H$. Der Mensch und sein Leib. Tübingen, Max Niemeyer Verlag, 1967.

15. Dworkin R R. Sovereign Virtue. Cambridge, London: Harvard University Press; 2000.

16. Mori M. The Twilight of "Medicine" and the Dawn of "Health Care". J Med Philosophy, 2000; 25: 723-744.

17. Satterwhite RC, Satterwhite WM \& Enarson C. An ethical paradox: the effect of unethical conduct on medical students' values. J Med Ethics 2000; 26:462-465.
18. Hébert PC, Meslin EM \& Dunn EV. Measuring the ethical sensitivity of medical students: a study at the University of Toronto. J Med Ethics 1992; 18:142-147.

19. Turner L. An anthropological exploration of contemporary bioethics: the varieties of common sense. J Med Ethics 1998; 24: 127-133.

20. Kottow MH. Vulnerability: what kind of principle is it? Medicine, Health Care and Philosophy. 2004; 7:281-287.

21. Grundstein-Amado R. Values education: a new direction for medical education. J Med Ethics 1995; 21: 174-178.

22. Kottow M, Bustos. Antropologia médica. Santiago: Ed. Mediterráneo; 2005.

\section{Endereço para Correspondência:}

Casilla 16168

Correo 9

Santiago, CHILE

e-mail: kottow@terra.com 